\title{
El desarrollo del juicio moral en Kohlberg como factor condicionante del rendimiento académico en ciencias sociales de un grupo de estudiantes de educación secundaria
}

\author{
Development of Moral Judgment according to Kohlberg as an Academic Performance \\ Determining Factor in Social Sciences for a Group of Secondary Education Students
}

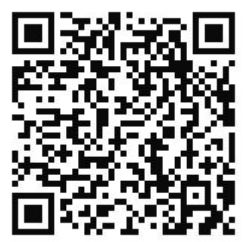

\author{
José Díaz-Serrano' \\ Universidad de Murcia \\ Facultad de Educación \\ Murcia, España \\ jose.d.s@um.es
}

Recibido 16 de setiembre de 2014 • Corregido 6 de julio de 2015 • Aceptado 31 de julio de 2015

\begin{abstract}
Resumen. Este estudio pretende determinar qué relación se produce entre el nivel de desarrollo moral del alumnado y su rendimiento académico en la asignatura de ciencias sociales-geografía, tomando como participantes a 12 estudiantes de tercer curso de educación secundaria obligatoria en un centro educativo de la ciudad de Murcia, España. Kohlberg teorizó un proceso de desarrollo del juicio moral en el que las personas pasamos por seis estadios, determinando en cada uno de ellos cómo afrontemos un dilema moral que se nos pueda plantear; un instrumento diseñado para esta investigación, basado en esta teoría, permitirá conocer el estadio de desarrollo moral de sus participantes. Los resultados serán reveladores de la existencia de una tendencia a que dicha relación sea directa y positiva, permitiendo concluir que resulta interesante atender al estadio de juicio moral en las aulas y a una serie de recomendaciones pedagógicas e investigadoras con las que concluye este artículo.
\end{abstract}

Palabras claves. Desarrollo moral, enseñanza centrada en el rendimiento, enseñanza de la geografía.

\footnotetext{
${ }^{1}$ Licenciado en Geografía; Máster Universitario en Formación del Profesorado de Educación Secundaria Obligatoria y Bachillerato, Formación Profesional, Enseñanza de Idiomas y Enseñanzas Artísticas; Máster Universitario en Investigación e Innovación en Educación Infantil y Educación Primaria; todos ellos por la Universidad de Murcia. Actualmente, inmerso en el Programa de Doctorado en Educación de la Escuela Internacional de Doctorado de la Universidad de Murcia, dentro de la línea "Investigación para la Mejora de la Educación en las Didácticas Específicas", es contratado predoctoral (FPU) en el Departamento de Didáctica de las Ciencias Matemáticas y Sociales y miembro del grupo de investigación de Didáctica de las Ciencias Sociales.
} 
doi: http://dx.doi.org/10.15359/ree.19-3.8

URL: http://www.una.ac.cr/educare

CORREO: educare@una.cr

\begin{abstract}
This study aims at defining the relation between the students' moral development level and their academic performance in the subject of Social Sciences-Geography, with the participation of twelve students enrolled in the third year of compulsory secondary education in school of Murcia, Spain. Kohlberg theorized the moral judgment development process in which people go through six stages, identifying in each one of them how we react when confronted with a moral dilemma; an instrument designed for this study, based on such theory, will allow us to know the stage of moral development of the participants. The results will reveal a tendency to a direct and positive relation, leading to the conclusion that it is interesting to examine the stage of moral judgment in the classroom. In addition, some educational and research recommendations are provided th the end of this paper.
\end{abstract}

Keywords. Moral development, proficiency-based teaching, Geography education.

En el año 1955, Lawrence Kohlberg empezó a realizar investigaciones sobre el razonamiento moral. Estas le llevaron a establecer que las personas utilizamos un proceso de desarrollo lógico de este similar al establecido por Piaget para el cognitivo. Cada persona posee unos valores que asimila de su entorno y actúa habitualmente de acuerdo con ellos, lo que se relaciona con la moralidad de la persona. Cuando esos valores entran en conflicto ante una situación determinada, que él concreta en problemas o dilemas morales, aparece el juicio o razonamiento moral; como señala Revenga (1992), en la persona se produce una crisis cuya solución implicará elegir entre adaptar el problema a su manera de pensar o acomodar su pensamiento para tratar de resolver los conflictos de acuerdo con su sistema de valores. Así, la práctica del juicio moral le hace reflexionar sobre sus propios valores morales y ordenarlos según una jerarquía lógica, haciéndolo de forma distinta dependiendo del estadio de desarrollo moral en el que se encuentre (Kohlberg, 1992).

Kohlberg establece seis estadios por los que toda persona va ascendiendo en el proceso de desarrollo del juicio moral que alcanza. En función del estadio moral en el que se encuentre cada sujeto, alcanzará a entender determinados razonamientos de distintas maneras, lo que posibilitará la comprensión, en mayor o menor medida, de determinadas explicaciones del profesorado que cuentan con cargas morales, impuestas por algunos de los contenidos de las asignaturas de ciencias sociales.

Desde la educación primaria, el Real Decreto 1513/2006 sobre enseñanzas mínimas en educación primaria en España establece, entre los objetivos de aprendizaje para el trabajo de la competencia social y ciudadana, el de "utilizar el juicio moral para elegir y tomar decisiones, y ejercer activa y responsablemente los derechos y deberes de la ciudadanía" (Ministerio de Educación y Ciencia [MEC], 2006, p. 43061). Sin embargo, en palabras de De Pro y Miralles (2009), este objetivo difícilmente se alcanzará a las edades del alumnado de educación primaria, ya que exigen "tener una experiencia, unos conocimientos y una conciencia de que existen diferentes perspectivas, recurrir a análisis multicausales y sistémicos, realizar razonamientos críticos y dialogar colectivamente escuchando, diferenciando ideas, tolerando las que no coinciden con las suyas..." (De Pro y Miralles, 2009, p. 71). Esta incapacidad no escapa al Real Decreto 
mencionado, que anuncia de antemano que su logro se producirá al final de la educación obligatoria; si bien, como señalan De Pro y Miralles (2009), el problema no es que nos digan qué se debe hacer al terminar la educación secundaria, sino la ausencia de pistas claras sobre los pasos a seguir en la primaria para hacerlos posibles más adelante. En este sentido, conocer el nivel de juicio moral del alumnado podría ser un buen punto de partida para trabajar mejor, no solo la competencia social y ciudadana, sino también los contenidos de ciencias sociales.

Este trabajo no solo parte de la idea de que el nivel de desarrollo moral que tenga el alumnado influirá en su comportamiento y forma de entender determinados sucesos que implican un razonamiento con cargas morales para su comprensión, sino que también presume que su rendimiento en la escuela puede estar influenciado por ese desarrollo moral, tratando de descubrir si esta relación es la clave que busca el profesorado de ciencias sociales-geografía para mejorar el bajo rendimiento que presenta un grupo de 12 estudiantes.

\section{Marco teórico}

La psicología cognitiva, en principio, relaciona lo moral con procesos cognitivos que explican y abordan el cómo y el porqué de su funcionamiento con un enfoque psicoanalítico y conductista (S. Palacios, B. Palacios y Ruiz, 2003). El modelo de razonamiento moral de Kohlberg ocuparía un papel hegemónico en el estudio del juicio y las justificaciones en asuntos sociomorales, gracias al importante trabajo realizado en la recogida de datos que justificasen su propuesta teórica, donde destaca la entrevista clínica de dilemas morales (Linde, 2010); además, estos estudios atenderían a otras dos necesidades: comprender y conocer las estrategias más adecuadas para favorecer el desarrollo moral y clasificar sujetos según su forma de aplicar principios morales a situaciones sociomorales (S. Palacios, B. Palacios y Ruiz, 2003).

El modelo de Kohlberg se centra en el conocimiento a través del desarrollo de reglas y la adquisición de principios universales. La moralidad es producto de la interacción creativa del sujeto con factores biológicos y culturales del medio en el que se inserta, junto con la adquisición de principios morales universales que se adquieren con la maduración del juicio cognitivo (Barra, 1987). Si Kohlberg no hubiera sido capaz de demostrar empíricamente que el desarrollo moral de las personas se caracteriza, en cada una de las seis etapas que establece, por los criterios que él asigna a cada una, no podría haber mantenido que sus estadios de juicio moral se fundamentan cognitivamente (Hersh, Reimer y Paolitto, 1984).

Elentendimiento socialymoral se desarrollajuntoconotrasformas del desarrollocognoscitivo y el contacto con el mundo físico estimula al niño y a la niña y le conduce al entendimiento de principios que debe respetar para vivir con otras personas y satisfacer sus aspiraciones como ser social, que podrán ser diferentes a las de otros individuos (Revenga, 1992). Kohlberg estableció como factores de una moral pronosticable la inteligencia, el conocimiento moral (reglas sociales), 
doi: http://dx.doi.org/10.15359/ree.19-3.8

URL: http://www.una.ac.cr/educare

CORREO: educare@una.cr

la tendencia a prever eventos, la capacidad para mantener una atención concentrada, la capacidad para controlar las fantasías no socializadas y la autoestima (Peters, 1984).

El desarrollo moral se da desde la niñez hasta la edad adulta, pasando por estadios que lo llevan de una moralidad heterónoma, en la que los patrones de comportamiento responden a normas y principios impuestos por un agente exterior, a una autónoma, en la que la determinación de la persona es libre y racional, porque se dota de un sistema normativo propio (Barra, 1987). Ante un problema moral, los sujetos se encuentran con el dilema de elegir entre la aplicación rigurosa de las reglas morales o la satisfacción y el bienestar de unos individuos en situaciones difíciles, siendo de interés, más que las soluciones dadas, las razones con las que justifican sus juicios morales (Rubio, 1987).

Al evaluar juicios morales y razonamientos de los individuos ante los dilemas morales, Kohlberg concluyó que en su desarrollo el ser humano pasa por tres niveles, a los que llamó preconvencional, convencional y postconvencional, dividiéndose cada uno en dos estadios (Linde, 2010); es decir, Kohlberg establece seis estadios o fases del juicio moral (véase tabla 1). El desarrollo moral consistirá en pasar de uno a otro, por orden, creciendo el nivel de moralidad con cada estadio superado (Quintana, 1995) y variando lo que una persona encuentra digno de valor en cada situación moral y por qué (Martínez, 2008). Además, los estadios son integraciones jerárquicas, de manera que el individuo comprende argumentos que pertenecen a su estadio y a uno por encima (Barra, 1987), siendo preciso hablar de estadio dominante (Rubio, 1987).

Tabla 1

Descripción de los niveles y estadios de desarrollo moral de Kohlberg

\begin{tabular}{ll}
\hline \multicolumn{1}{c}{ Niveles } & \multicolumn{1}{c}{ Estadios } \\
\hline $\begin{array}{l}\text { PRECONVENCIONAL (individualista y coercitivo): aún } \\
\text { no entiende las normas sociales convencionales y se } \\
\text { respetan para evitar el castigo. }\end{array}$ & $\begin{array}{l}\text { ESTADIO 1: moral de la obediencia y del castigo. } \\
\text { ESTADIO 2: bien como satisfacción de necesidades, } \\
\text { personales y ajenas. }\end{array}$ \\
$\begin{array}{l}\text { CONVENCIONAL (social, razonable): se somete a } \\
\text { reglas y expectativas de la sociedad y la autoridad y } \\
\text { las defiende. }\end{array}$ & $\begin{array}{l}\text { ESTADIO 3: relaciones interpersonales buscan } \\
\text { satisfacer las expectativas mutuas. }\end{array}$ \\
$\begin{array}{ll}\text { ESTADIO 4: el respeto a la ley y al orden, a la } \\
\text { Convivencia y a la conciencia. }\end{array}$ \\
$\begin{array}{l}\text { entiende y acepta normas que apoyan unos } \\
\text { principios morales. }\end{array}$ & ESTADIO 5: moral del contrato social. \\
\hline
\end{tabular}

Nota: Elaboración propia, a partir de las aportaciones de Linde (2010) y Quintana (1995).

4 
En los últimos años, numerosos estudios se han dedicado a la validación o adaptación de pruebas para medir estadios de desarrollo moral, poniendo a prueba en el proceso hipótesis que relacionan el desarrollo moral con aspectos como el desarrollo político y religioso, el desarrollo profesional, los procesos de democratización, ideologías políticas, la comprensión internacional o la trasgresión de los símbolos nacionales (S. Palacios, B. Palacios y Ruiz, 2003). Asimismo, han proliferado las investigaciones sobre implicaciones docentes que están en la base del desarrollo del juicio moral, destacando la experiencia de Narváez y Gleason (2007) al estudiar la influencia que tiene la relación entre desarrollo moral y experiencia educativa sobre la memorización de textos con cargas morales; así como la tesis doctoral de Lewis (2007), centrada en la evaluación del desarrollo moral en estudiantes con altas capacidades.

La educación moral consiste en promover que el alumnado vaya ascendiendo por los estadios, lo que se presta a un diseño educativo por objetivos (Quintana, 1995). Esta progresión no deriva de lo que enseñan las personas adultas (Peters, 1984), pero puede ser impulsada por una estimulación cognoscitiva que ayude a establecer una discrepancia óptima entre lo que el niño o la niña ha dominado ya y lo que debe dominar (Kohlberg, 1992).

Parte del contenido de la moral se transmite mediante el ejemplo y la instrucción, pero la forma debe ser entendida por el propio individuo mediante la estimulación apropiada en situaciones concretas (Peters, 1984). No hay unas cualidades morales o hábitos ("virtudes") en los que haya que formarse; de lo contrario, la educación moral consistiría en practicar y formar en ellos al alumnado. Normalmente, esas virtudes son etiquetas que la gente alaba o censura, lo que no coincide con la toma de decisiones morales (Quintana, 1995).

Para que un alumno vea algo propio de un estadio, el profesor debe llamar la atención hacia características de los sucesos y esperar que se abra la puerta; pero no puede hacer que memorice un contenido o practique un movimiento, como al impartir información o habilidad (Peters, 1984). Además, el discurso no debe orientar contenidos, sino un conglomerado de presupuestos que garantice la imparcialidad en la formación del juicio (Habermas, 2000).

\section{Objetivos}

Los objetivos a los que trata de dar respuesta este trabajo tienen como propósito principal determinar si el nivel de desarrollo moral del grupo de estudiantes participantes tiene influencia alguna sobre su rendimiento académico en la asignatura de ciencias sociales-geografía en educación secundaria obligatoria. Este objetivo principal se concreta en los siguientes objetivos específicos:

1. Determinar el estadio de desarrollo moral de Kohlberg en el que se encuentran el alumnado participante y extraer conclusiones en relación con el género y la edad.

2. Relacionar el estadio de desarrollo moral estudiantil con sus resultados académicos en ciencias sociales, medidos en nota media, para descubrir en qué medida se correlacionan ambas variables. 
doi: http://dx.doi.org/10.15359/ree.19-3.8

URL: http://www.una.ac.cr/educare

CORREO: educare@una.cr

\section{Metodología}

\section{Diseño}

Este estudio adopta un diseño analítico cuantitativo de grupo único, utilizando una metodología ex-post-facto, en la que el investigador no tiene un control directo sobre las variables independientes o predictoras, en cuanto que ya acontecieron sus manifestaciones o porque no pueden ser manipulables intrínsecamente; esto es, se hacen inferencias sobre las relaciones, a partir de la variación común de las variables independientes y dependientes, sin intervención directa (Arnal, Del Rincón y Latorre, 1992; Ramos, 2011). Además, se aborda con un carácter exploratorio y la finalidad de establecer una primera aproximación al área problemática planteada, en cuanto a que el uso de variables, siendo una condición necesaria en la investigación ex-post-facto, su relación significativa no sea suficiente para poner de manifiesto las relaciones de causalidad (Latorre, Del Rincón y Arnal, 2005). En este estudio, las variables independientes serán género y nota media del expediente, mientras que el estadio de desarrollo moral será la variable dependiente.

\section{Participantes y contexto}

Los participantes de esta investigación fueron 12 alumnos (seis niños y seis niñas) del tercer curso de educación secundaria obligatoria de un centro público ubicado en el sur de la capital murciana. En general, el nivel académico de los padres es medio-alto y la estabilidad laboral elevada. Aunque no hay grupos considerables de población desfavorecida, se atienden estudiantes que, por su origen o ambiente socio-familiar, precisan atención compensatoria, este es un grupo muy heterogéneo que registra bajo rendimiento en la asignatura de ciencias sociales-geografía. Así, la selección de los participantes responde a un muestreo intencional para controlar lo más posible el efecto de la calificación académica; respetándose el procedimiento de grupo intacto, a posteriori se seleccionaron tres estudiantes de mayor nota media y tres que la tenían más baja para cada género.

\section{Recogida de información: instrumento y procedimiento}

Antes de comenzar con el estudio, el profesor procedió a la obtención de los permisos paternos de participantes, así como de la dirección del centro, indicándoles la información a recabar, el objeto del estudio y el tratamiento y uso que se haría de los datos. Contando con los permisos de ambas partes, se diseñó un instrumento utilizando una adaptación del dilema número tres de Kohlberg, a partir del utilizado por Revenga (1992) y Hersh et al. (1984). 
El instrumento de recogida de información se entregó a todos los estudiantes del grupo, asegurándoles el anonimato y la confidencialidad de la información, animándoles a participar por la importancia de sus aportaciones para la investigación. Respondieron de forma independiente y autónoma en una sesión única.

\section{Plan de análisis de la información}

El estadio que caracteriza el juicio moral del estudiantado, se constituye como una variable cuantitativa, sin embargo, el resultado es fruto del análisis cualitativo de las justificaciones que aportan los alumnos alumnas al responder al dilema moral planteado, utilizando la herramienta ATLAS.ti Qualitative Data Analysis (versión 6.2) y fundamentando el proceso inductivo en una elaboración previa de categorías que, con base en la teoría revisada y categorizaciones de investigaciones anteriores, definen cada uno de los seis estadios. Por su parte, el análisis cuantitativo de la información se ha realizado con el paquete estadístico IBM-SPSS Statistic (versión 19), aplicándose estadísticos descriptivos, tablas de contingencia y pruebas de asociación ji-cuadrado de Pearson o Rho de Spearman.

\section{Desarrollo de los resultados}

El análisis de los datos recogidos de acuerdo con el diseño anteriormente descrito ha culminado en la obtención de unos resultados cuyo desarrollo se estructura atendiendo a los objetivos del estudio que se presentaban en este trabajo.

\section{Determinar el estadio de desarrollo moral de Kohlberg en el que se encuentran los alumnos participantes y extraer conclusiones en relación con el género y la edad}

Los estudiantes participantes se encuentran predominantemente en el tercer estadio de desarrollo moral, representando este la mitad de los estudiantes (6 alumnos), frente a los cuatro estudiantes que se encuentran en el cuarto estadio y los dos que están en el quinto. Sin embargo, el análisis del desarrollo moral de los participantes por género, arroja diferencias importantes, encontrando fases más avanzadas en los hombres que en las mujeres; mientras que los hombres se distribuyen a partes iguales entre los estadios 3, 4 y 5 , las mujeres se encuentran mayoritariamente en el estadio 3 , algunas en el cuarto y no tienen representación en el quinto (véase tabla 2), aunque la prueba ji-cuadrado de Pearson no revela una asociación estadísticamente significativa entre las variables de estadio de desarrollo moral del estudiante y su género $[x 2(2)=2, p=.368]$ a un nivel crítico $a=.05$. 
doi: http://dx.doi.org/10.15359/ree.19-3.8

URL: http://www.una.ac.cr/educare

CORREO: educare@una.cr

Tabla 2

Estadio de desarrollo moral de Kohlberg, según género de estudiantes

\begin{tabular}{lccc}
\hline Estadio de desarrollo moral & Hombre & Mujer & Total \\
\hline Estadio 3 & 2 & 4 & 6 \\
Estadio 4 & 2 & 2 & 4 \\
Estadio 5 & 2 & 0 & 2 \\
\hline Total & 6 & 6 & 12 \\
\hline
\end{tabular}

Nota: Elaboración propia.

En relación con la edad, la totalidad tiene la correspondiente al tercer curso de la educación secundaria obligatoria que están cursando, es decir, 14015 años. Ante la presencia de repitientes que aún no han cumplido años en el presente curso y otros que cuentan 15 años alcanzados en el año en curso, se considera rechazar posibles resultados detectados que relacionan el estadio de desarrollo moral con la edad.

\section{Relacionar el estadio de desarrollo moral de estudiantes con sus resultados académicos para descubrir si existe una correlación entre ambas variables}

La aplicación del índice de correlación de Spearman está justificada doblemente: se pretenden correlacionar dos variables ordinales y una muestra inferior a 30 sujetos avala una prueba estadística no paramétrica. El cálculo de la Rho de Spearman entre las variables estadio de desarrollo moral y nota media de las dos primeras evaluaciones arroja un coeficiente que indica una relación media, con signo positivo y estadísticamente no significativa $\left(R_{R}=.487, p=.108\right)$ a un nivel crítico $a=.05$, reflejando que las variables correlacionan de forma moderada y positiva, es decir, existe una tendencia a que cuanto mayor es el estadio de desarrollo moral, los resultados académicos alcanzan valores más altos.

\section{Conclusiones, consecuencias e implicaciones}

Con este trabajo empírico se ha intentado descubrir, describir y comprender el nivel de desarrollo moral que, siguiendo las aportaciones de Lawrence Kohlberg, caracteriza a un grupo de estudiantes de ciencias sociales-geografía del tercer curso de educación secundaria obligatoria, según su género, así como la influencia que tiene este sobre su rendimiento académico. Las conclusiones más importantes a las que se hallegado son: 
- La mayoría de participantes de este estudio se encuentran en un estadio dominante 3 de desarrollo moral, aunque también tienen amplia representación en el 4.

- Pese a que la asociación no es estadísticamente significativa, el desarrollo moral en los chicos participantes alcanza estadios más altos que en las chicas.

- Aunque no aparece una correlación estadísticamente significativa, existe una tendencia media a que los resultados académicos en ciencias sociales sean mejores cuando el estadio de juicio moral del estudiantado es más alto.

La adolescencia puede dividirse en una temprana entre los 11 y 14 años, una media entre los 15 y los 18 años y una adolescencia tardía a partir de los 18 años (Moreno, 2010). El sujeto preadolescente o adolescente sufre cambios cognitivos y de rol social que marcan la entrada en el nivel de razonamiento moral convencional, mientras que el paso al estadio 4 suele comenzar a mitad de la adolescencia (Hersh et al., 1984), lo que justifica que quienes participan de este estudio, de entre 14 y 15 años, en el paso a la adolescencia media, se encuentren en dicha transición y, por ello, mayoritariamente en los estadios 3 y 4.

El paso al estadio 3 de desarrollo moral implica cambiar de la posición social de intereses individuales a la de los grupales o de la sociedad. Además, adoptará la perspectiva de terceras personas, permitiéndole percibir cómo reaccionará el grupo ante sus actos. En el estadio 4, los valores de la ley y la vida entran en conflicto y las personas tienen un problema en elegir entre ellos (Hersh et al., 1984), lo que lleva a Kohlberg (1992) a ver inadecuada esta etapa para resolver situaciones en una sociedad cuyo sistema legal niega derechos humanos básicos; situaciones que sí que podrán afrontar los dos estudiantes del estadio postconvencional, aventajados moralmente hablando, ya que, cuando se alcanza, rara vez se hace antes de los 20 años (Hersh et al., 1984), pese a que Kohlberg ya detectó adolescentes que utilizaban este razonamiento moral junto con estadios inferiores.

El estadio de desarrollo moral en función del género arroja la conclusión de que este, en los hombres de la muestra, alcanza niveles más altos que en las mujeres. Esto también se ha constatado en otros estudios y ha sido origen de críticas al test de Kohlberg. Esta situación, según Gilligan (1994), responde, entre otros motivos, a que las características cognitivas de la mujer son distintas a las del hombre y les llevan a ser más capaces de sentir empatía; si estas cualidades dejaran de ser vistas como deficiencias para verse como componentes esenciales del razonamiento moral adulto, lo que parece una confusión moral de las mujeres, pasaría a ser un signo de fortaleza. Sin embargo, Kohlberg (1992) justifica estas diferencias por género diciendo que el papel de la mujer en el hogar limitaba su contacto con el entorno social y, con ello, su desarrollo moral, pero esta teoría en la actualidad se desmonta en gran medida; más aún en edades como las del grupo participante en este estudio. 
doi: http://dx.doi.org/10.15359/ree.19-3.8

URL: http://www.una.ac.cr/educare

CORREO: educare@una.cr

Los resultados de esta investigación aportan evidencias para apoyar un modelo educativo que favorezca el desarrollo moral estudiantil como vía de mejora del rendimiento académico, al menos en ciencias sociales-geografía, lo que exige introducir cambios en las formas actuales de interacción entre docentes, estudiantes y centro escolar.

El hecho de que el individuo solo comprenda los argumentos morales de su estadio y uno superior (Barra, 1987) da sentido a que el desarrollo de este influya en su rendimiento académico. Cabe suponer que los argumentos morales utilizados por el profesorado para justificar determinados sucesos solo podrán ser abarcados por estudiantes que tengan el desarrollo moral que el profesorado, al construir sus explicaciones, estime que alcanzan; para adecuar las explicaciones con implicaciones morales a las capacidades de sus estudiantes, sería de gran utilidad una evaluación destinada a conocer su nivel de desarrollo moral.

El desarrollo moral va unido al de la estructura cognitiva, mediante la interacción entre la estructura del organismo y la del medio en el que vive el sujeto, especialmente el social. El conocimiento social requiere asumir el rol de la otra persona; los cambios evolutivos del yo social se producirán a la vez en las concepciones del mundo social (Kohlberg, 1992), concepciones que determinarán cómo comprenda cada estudiante los aspectos sociales que marcan los hechos históricos, geográficos o artísticos del currículo de las asignaturas de ciencias sociales en educación secundaria, así como la mayor o menor profundidad con que lo haga.

Antes de atender las prescripciones educativas relacionadas con el desarrollo moral, es importante atender las consideraciones de Habermas (2000), que apunta que la ética discursiva debe garantizar la imparcialidad en la formación del juicio, y a las palabras del propio Kohlberg (1992) que afirman que la educación moral no procura transmitir contenidos, sino capacitar facultades. La libertad de los educandos solo quedará protegida en un modelo de educación moral relacionado con la estimulación y que respete la autonomía del niño y la niña; de lo contrario, se estaría cayendo en la indoctrinación (Quintana, 1995). Las asignaturas de ciencias sociales han de dar a conocer la pluralidad política, económica y social pasada y actual, contenidos que deben impartirse sin que dominen las opciones elegidas por el profesorado; aunque afloran irremediablemente, pienso, no han de hacerlo como adoctrinamiento.

Como se decía, el personal docente debe conocer el nivel de desarrollo moral de sus estudiantes, pero también lo que fomenta su crecimiento, es decir, la creación de un ambiente de clase que asegure el desarrollo moral (Palomo, 1989). La educación no puede ser ajena a la moral, lo que implica modificar la estructura actual de las clases para lograr la participación democrática estudiantil, básica para la maduración del sentido ético (Quintana, 1995). Esto es lo que Kohlberg (1992) Ilama método de enseñanza ética, a lo que añade el diseño de una forma de escuela que asemeje la vida escolar a una comunidad justa con una estructura democrática de participación, en la que el derecho a la libertad de cada quien sea compatible con el de los demás individuos y en la que reine la imparcialidad y reversibilidad. 
doi: http://dx.doi.org/10.15359/ree.19-3.8

URL: http://www.una.ac.cr/educare

CORREO: educare@una.cr

El desarrollo moral de la niñez termina dándole el criterio de justicia, mientras que la moralización del individuo y el centro educativo consiste en que vivan según el ideal de justicia; esto exige que funcionen de forma democrática (Quintana, 1995). Linde (2010) recoge las condiciones que deben caracterizar la atmósfera del centro escolar para una mayor influencia sobre el juicio y la conducta morales, mientras que Hersh et al. (1984) hacen algunas aportaciones útiles para orientar al profesorado en la creación de un clima de clase que facilite el desarrollo moral; ambas aportaciones se sintetizan en la tabla 3.

Tabla 3

Condiciones que deben caracterizar la atmósfera del centro escolar (Linde, 2010) y el clima de clase (Hersh et al., 1984) para el desarrollo del juicio y la conducta morales

\begin{tabular}{|c|c|}
\hline Centro escolar & Aula \\
\hline $\begin{array}{l}\text { - Discusión abierta centrada en la justicia y la moral. } \\
\text { - Participación en la creación de reglas y el ejercicio } \\
\text { del poder y la responsabilidad. } \\
\text { - Conflicto cognitivo estimulado por el contacto con } \\
\text { otros puntos de vista. } \\
\text { - Desarrollo comunitario hacia etapas morales } \\
\text { superiores. }\end{array}$ & $\begin{array}{l}\text { - Distribución en grupos pequeños para facilitar la } \\
\text { apertura estudiantil. } \\
\text { - Disposición de aula que facilite debate entre } \\
\text { ayudiantes y la comunicación con su docente, } \\
\text { expresivos oralmente. } \\
\text { - Proponer modelos de aceptación: una atmósfera } \\
\text { de clase que no juzgue y fomente opiniones } \\
\text { abiertas. } \\
\text { - Técnicas de escucha y comunicación. } \\
\text { - Animar la interacción entre estudiantes, para que } \\
\text { los niños y las niñas busquen aprender y pensar } \\
\text { confrontando ideas. }\end{array}$ \\
\hline
\end{tabular}

Nota: Elaboración propia.

Todas las técnicas para favorecer el desarrollo moral no han de darse exclusivamente por separado, sino que es útil ponerlas en práctica de forma integrada en las conductas de cada educador (Palomo, 1989). Una forma de aplicación en el aula puede ser el planteamiento de dilemas morales hipotéticos o reales, invitándoles a buscar la solución. Un ejemplo para ciencias sociales, planteado por Quintana (1995), es el que debió plantearse el presidente Truman durante la Segunda Guerra Mundial para decidir si lanzaba la bomba atómica, donde la interrogación guiada, la explicación de opiniones, el posicionamiento en el papel de los personajes (role-play) o los argumentos morales superiores del personal docente serán de utilidad.

Esta educación no se nota en el conocimiento o el comportamiento generado, sino en la capacidad para participar en el proceso social (Quintana, 1995), y es más fácil evaluarla en una comunidad escolar de participación democrática como la que planteaba Kohlberg. 
doi: http://dx.doi.org/10.15359/ree.19-3.8

URL: http://www.una.ac.cr/educare

CORREO: educare@una.cr

La educación moral ayudará a lograr que los niños y las niñas aprendan las destrezas críticas para una ciudadanía global (Narváez, 2003). Cualquier día, a cualquier hora y en cualquier nivel, se enfrentan con aspectos morales como hacer trampas, mentir, obedecer, vengarse, respetar la amistades, ... y el ambiente social de un centro escolar permite utilizar contenidos para ayudarlas en el desarrollo moral de su conducta (Palomo, 1989). En el caso de las ciencias sociales, considero que son numerosos y, como demuestra esta investigación, si se aprovechan para fomentar el desarrollo moral estudiantil el profesorado estará, a su vez, contribuyendo a la mejora del rendimiento académico de sus pupilos y pupilas.

Como mejoras para el futuro, es necesario ampliar mucho más las investigaciones en el sentido de este trabajo para poder confirmar en mayor medida las conclusiones alcanzadas, especialmente dilatando el número de sujetos estudiados. Además, sería importante determinar si el desarrollo moral alcanza un papel más determinante para el rendimiento académico estudiantil en ciencias sociales que en otras asignaturas, para lo que es necesario analizar comparativamente su rendimiento en las diversas asignaturas que cursa. Por último, sería interesante evaluar la mejora en los resultados de aprendizaje de un grupo de estudiantes, tras la implementación a lo largo de un curso de propuestas didácticas basadas en el desarrollo moral de sus estudiantes.

\section{Referencias}

Arnal, J., Del Rincón, D. y Latorre, A. (1992). Investigación educativa. Fundamentos y metodología. Barcelona: Labor.

Barra, E. (1987). El desarrollo moral: Una introducción a la teoría de Kohlberg. Revista Latinoamericana de Psicología, 19(1), 7-18. Recuperado de http://www.redalyc.org/ articulo.oa?id=80519101

De Pro, A. y Miralles, P. (2009). El currículo de conocimiento del medio natural, social y cultural en educación primaria. Educatio Siglo XXI, 27(1), 59-96. Recuperado de http://revistas.um.es/ educatio/article/view/71101/68641 Gilligan, C. (1994). La moral y la teoría: Psicología del desarrollo femenino. México: Fondo de Cultura Económica.

Habermas, J. (2000). Conciencia moral y acción comunicativa. Barcelona: Península.

Hersh, R., Reimer, J. y Paolitto, D. (1984). El crecimiento moral de Piaget a Kohlberg. Madrid: Narcea Ediciones.

Kohlberg, L. (1992). Psicología del desarrollo moral. Bilbao: Desclée de Brouwer.

Latorre, A., Del Rincón, D. y Arnal, J. (2005). Bases metodológicas de la investigación educativa. Barcelona: Ediciones Experiencia. 
Lewis, A. E. (2007). The Moral Judgment of Gifted Adolescents [El juicio moral de los adolescentes superdotados] (Tesis doctoral). University of Minnesota. Recuperado de http://books. google.es/books?id=Py9AIQQ9TIgC\&pg

Linde, A. (2010). Síntesis y valoración de la teoría sobre el desarrollo moral de Lawrence Kohlberg, Ágora, 29(2), 31-54. Recuperado de http://dspace.usc.es/bitstream/10347/7373/1/pg 032055 agora29-2.pdf

Martínez, J. G. (2008). Desarrollo moral: Su medición y factores determinantes en un grupo de estudiantes de décimo grado (Tesis de maestría). Universidad Sergio Arboleda, Bogotá. Recuperado de http://www.usergioarboleda.edu.co/civilizar/ invedusa/desarrollo-eticoestudiantes.pdf

Ministerio de Educación y Ciencia (MEC). (8 de diciembre de 2006). Real Decreto 1513/2006, de 7 de diciembre por el que se establecen las enseñanzas mínimas de la educación primaria. BOE, núm. 293, 43053-43102. Recuperado de http://www.boe.es/boe/dias/2006/12/08/ pdfs/A43053-43102.pdf

Moreno, A. (2010). Los estudiantes de educación secundaria: Características y contextos de desarrollo y socialización. En C. Coll (Coord.), Desarrollo, aprendizaje y enseñanza en la educación secundaria (pp.11-29). Barcelona: Graó.

Narváez, D. (2003). Educación y desarrollo moral. Republicana Política y Sociedad, 2(2), 39-49. Recuperado de http://148.202.18.157/sitios/publicacionesite/pperiod/republicana/pdf/ ActaRep02/articulos39.pdf

Narváez, D. y Gleason, T. (2007). The Relation of Moral Judgment Development and Educational Experience to Recall of Moral Narratives and Expository Texts [La relación del desarrollo del juicio moral y la experiencia educativa de recordar las narrativas morales y los textos expositivos]. The Journal of Genetic Psychology, 168(3), 251-276. doi: http://dx.doi. org/10.3200/GNTP.168.3.251-276

Palacios, S., Palacios, B. y Ruiz, S. (2003). Nuevas formas de medir el desarrollo sociomoral. Revista de Psicodidáctica, 14, 41-58. doi: http://dx.doi.org/10.1387/RevPsicodidact.143

Palomo, A. M. (1989). Laurence Kohlberg: Teoría y práctica del desarrollo moral en la escuela. Revista Interuniversitaria de Formación del Profesorado, 4, 79-90. Recuperado de http:// dialnet.unirioja.es/descarga/articulo/117615.pdf

Peters, R. S. (1984). Desarrollo moral y educación moral. México: Fondo de Cultura Económica. 
doi: http://dx.doi.org/10.15359/ree.19-3.8

URL: http://www.una.ac.cr/educare

CORREO: educare@una.cr

Quintana, J. M. (1995). Pedagogía moral. El desarrollo moral integral. Madrid: Dykinson.

Ramos, J. L. (2011). Investigación cuasiexperimental. En S. Cubo, B. Martín y J. L. Ramos (Coords.), Métodos de investigación y análisis de datos en ciencias sociales y de la salud (pp. 329-371). Madrid: Pirámide.

Revenga, M. (1992). Percepción de la educación familiar y desarrollo del razonamiento moral en el adolescente (Tesis doctoral). Recuperada de http://biblioteca.ucm.es/tesis/19911996/S/4/ S4002001.pdf

Rubio, J. (1987). El hombre y la ética. Humanismo crítico, desarrollo moral, constructivismo ético. Barcelona: Anthropos.

\section{Cómo citar este artículo en APA:}

Díaz-Serrano, J. (Setiembre-Diciembre, 2015). El desarrollo del juicio moral en Kohlberg como factor condicionante del rendimiento académico en ciencias sociales de un grupo de estudiantes de educación secundaria. Revista Electrónica Educare, 19(3), 1-14. doi: http://dx.doi.org/10.15359/ree.19-3.8

Nota: Para citar este artículo en otros sistemas puede consultar el hipervínculo "Como citar el artículo" en la barra derecha de nuestro sitio web: http://www.revistas.una.ac.cr/index.php/EDUCARE/index 